\title{
Both Aerobic and Resistance Exercise Ameliorate Nonalcoholic Fatty Liver Disease Even in the Absence of Weight Reduction in High-fat Diet- induced Obese Mice
}

Ju Yong Bae ( $\sim$ kosa99@dau.ac.kr)

College of Arts and Physical Education, Dong-A University

Research

Keywords: Aerobic exercise, lipolytic enzyme, Hepatic steatosis, CB1 receptor, NAFLD

Posted Date: August 25th, 2020

DOI: https://doi.org/10.21203/rs.3.rs-61507/v1

License: (9) This work is licensed under a Creative Commons Attribution 4.0 International License. Read Full License 


\section{Abstract}

Background: This study aimed at analyzing how aerobic and resistance exercise without dietary restriction could affect fat accumulation in the liver of high-fat diet (HFD)-induced obese mice.

Methods: Mice, divided into normal (CO) and high-fat diet (HF) groups, were fed normal or $60 \%$ HFD diets for 8 weeks, respectively. The HF group was then subdivided into HFD + sedentary (HF), HFD + aerobic training (HFAT), and HFD + resistance training (HFRT) groups. The mice in the training groups underwent treadmill or ladder climbing training for 8 weeks.

Results: Although the HFAT and HFRT group body weight did not decrease, fat mass significantly decreased in the training groups than that in the HF group. Liver triglyceride levels in the HF group significantly increased compared to the $\mathrm{CO}$ group while no significant difference was detected in the training groups. HFD significantly induced an increase of liver cannabinoid-1 receptor, Sterol-regulatory element binding protein 1 (SREBP-1), and fatty acid synthase. Both aerobic and resistance training significantly induced the increase of liver carnitine palmitoyltransferase1, phosphor AMP-activated protein kinase ( $p$-AMPK), and p-AMPK/AMPK while inducing a decrease of liver SREBP-1. However, the type of exercise did not cause any significant difference.

Conclusions: Both aerobic and resistance exercise effectively improved fat mass and NAFLD even without weight reduction in HFD-induced obese mice. Therefore, a selected sustainable type of exercise based on the fitness level, type of disease, musculoskeletal disorders status, and preference of subjects, would be the best exercise intervention for alleviating NAFLD.

\section{Background}

Consumption of chronic High-fat diets (HFD) increases body weight and fat mass, which is a well-known powerful factor in causing obesity. The excess nutrients from dietary intake are stored in the form of lipid droplets in the adipose tissue but lipids that exceed the storage capacity of the adipose tissue lead to increased fat accumulation in other tissues, including the liver, muscle, and heart [1,2]. The accumulation of excessive lipids in non-adipose tissues could lead to cell dysfunction, lipotoxicity, and cell death [3].

Currently, non-alcoholic fatty liver disease (NAFLD) is closely associated with obesity [4] and emerged as the most common chronic liver disease [5]. The prevalence of NAFLD in the Asian population is around $25 \%$, which rate is similar to many western countries [6]. Although hepatocellular carcinoma and endstage liver disease secondary to NAFLD rarely occur, it has shown a rising trend [7] along with the increase in mortality [8].

Bodyweight reduction of at least $3-5 \%$ is essential to alleviate NAFLD as the occurrence of NAFLD is closely related to obesity and increased body fat mass $[9,10]$. Therefore, lifestyle modifications such as exercise and dietary restriction have been recommended as the most effective intervention of NAFLD as such powerful interventions could reduce bodyweight [11]. Especially, aerobic exercise is a common 
exercise treatment for attenuating the NAFLD because it has beneficial effects for weight loss by increasing energy consumption. $[9,12]$.

However, the results of animals and human studies suggested that regular exercise might be effective in alleviating NAFLD even if weight loss does not be induced. Some previous studies reported that exercise without dietary or caloric restriction did not affect weight loss $[13,14]$. Although there was no benefit of weight reduction, regular aerobic exercise increased lipolytic proteins in the liver and improved glucose tolerance in HFD-induced obese mice [13]. A Previous human study also reported that four weeks of aerobic exercise led to a decrease in hepatic triglyceride concentration and visceral adiposity in obese individuals without weight loss [14]. In this respect, although resistance exercise is not an effective strategy for weight loss, I thought that resistance exercise through repetitive muscle contraction and relaxation could have a positive effect on histological changes in liver tissue. Besides, in the case of obesity, the effect of exercise mainly occurs when the intervention is done with dietary restriction, but the effect of aerobic or resistance exercise alone on lipolysis of the liver tissue is not clear.

Therefore, the purpose of this study was to analyze the effects of aerobic and resistance exercise without dietary restriction on fat accumulation in the liver tissue in high-fat diets induced obese mice.

\section{Methods}

Animals and maintenance

Forty male C57BL/ 6 (4 weeks old) mice were purchased from Samtako Bio Korea (Osan, Korea) and fed freely with standard chow and water until 30 weeks old. Three or four mice were housed in each cage and maintained under standardized conditions in an animal facility of the Dong-A University College of Medicine. Relative humidity ( $55 \pm 5 \%)$, temperature $\left(22 \pm 2{ }^{\circ} \mathrm{C}\right)$ and a 12-h dark-light cycle remained constant. The animal experiments were approved by the Dong-A University Medical School Institutional Animal Care and Use Committee (DIACUC-approval-19-11) and all procedures were conducted in accordance with the committee guidelines.

Experimental design

When the mice were 30 weeks old, mice were randomly divided into two groups to induce obesity by HFD: a normal diet $(\mathrm{CO}, \mathrm{n}=10)$ and a high-fat diet $(\mathrm{HF}, \mathrm{n}=30)$ groups. The HF group was fed with a $60 \% \mathrm{fat}$ chow (60\% lipid, $20 \%$ carbohydrate, and $20 \%$ protein), whereas the CO group was fed with standard chow (6.3\% lipid, $69.4 \%$ carbohydrate, and $24.3 \%$ protein) for eight weeks. Following obesity induction period, the mice in the HF group were randomly subdivided into an HFD + sedentary (HF, $n=10), H F D+$ aerobic training (HFAT, $n=10$ ), and HFD + resistance training (HFRT, $n=10$ ) groups, and the training groups exercised for eight weeks. Body weight was measured every week until the end of the experimental period.

Training intervention 
Mice in both aerobic and resistance training groups exercised with a $65 \sim 70 \%$ of exercise intensity for 8 weeks, five days per week while maintaining an HFD. In the first week, mice exercised at a low intensity for adaptation of the exercise equipment and to set their exercise intensity. Thereafter, mice exercised at a determined speed or weight for the remaining 7 weeks. Mice in the $\mathrm{CO}$ and HF groups were placed next to the treadmill to expose a similar stress condition when the mice in the training groups were exercising.

Mice in the HFAT group exercised 5 days per week for 8 weeks using an animal treadmill. During the four days of the first week, aerobic exercise was performed for $5 \mathrm{~min}$ at a speed of $5 \mathrm{~m} / \mathrm{min}$ on the first day, and the exercise time was increased 5 min every day. On the 5th day of the first week, an incremental load test was performed to determine the treadmill exercise speed of the HFAT group [15]. Briefly, after 5 min warm-up at $5 \mathrm{~m} / \mathrm{min}$, the speed of the treadmill was increased by $3 \mathrm{~m} / \mathrm{min}$ every 3 min at $0 \%$ grade until exhaustion. The exercise speed in the HFAT was set to $65 \sim 70 \%$ of the maximal speed based on the results of the incremental load test. Mice exercised 5 days per week, 50 minutes a day, including 10 min of warm-up and 10 min of cool-down exercise.

Mice in the HFRT group exercised 5 days per week for 8 weeks using a climbing ladder $(100 \mathrm{~cm}$ ladder with $2 \mathrm{~cm}$ grid and $80^{\circ}$ incline). House Chamber $(\mathrm{L} \times \mathrm{W} \times \mathrm{H}=25 \times 25 \times 20 \mathrm{~cm})$ was set up on the top of the ladder for a 2-min rest between the trial. During the four days of the first week, exercise was performed without any resistance for adaptation of the ladder. On the 5th day of the first week, an incremental load test was conducted by referring to the previous study to determine the exercise intensity [16]. Briefly, 50\% of body weight was attached to the mouse's tail (lead), and the lead weight was progressively increased by $3 \mathrm{~g}$ when the mouse was a success to climb the ladder. Mice exercised 8 sets for the remaining 7 weeks with a $65 \sim 70 \%$ of the maximum successful weight. An incremental load test was conducted after 4 weeks to adjust the weight of the load.

Tissue sampling

Tissue sampling was conducted two days after the last exercise session to rule out temporary training effects. Food was removed $12 \mathrm{~h}$ before sacrifice. The liver samples were excised after complete anesthesia using ethyl ether. The samples were immediately frozen in liquid nitrogen and stored at $-80{ }^{\circ} \mathrm{C}$ refrigerator.

Hematoxylin and eosin staining

Small pieces of liver tissue were fixed with formalin ( $10 \%$ neutral-buffer formalin) and embedded in paraffin. Four-micrometer sections were cut and stained using hematoxylin and eosin (H\&E staining). Digital images of the slides were captured with an Aperio ScanScope (Leica Biosystems, Buffalo Grove, IL, USA).

\section{Extraction of liver triglycerides}

As previously described [13], Liver tissue (50 mg) was placed in $200 \mu$ l of ethanolic $\mathrm{KOH}$ (2 parts ethanol: 1 part $30 \% \mathrm{KOH}$ ) and incubated overnight at $55{ }^{\circ} \mathrm{C}$. Subsequently, it was mixed with $50 \%$ ethanol into 
each tube to bring the volume to $0.5 \mathrm{ml}$ and centrifuged for $5 \mathrm{~min}$ at 13,000 rpm. The supernatant was transferred into new tubes, and $50 \%$ ethanol was added again into each tube to bring the volume to 0.6 $\mathrm{ml}$. After vortexing, $200 \mu \mathrm{l}$ was moved to a new tube, and $215 \mu \mathrm{l}$ of $1 \mathrm{M} \mathrm{MgCl} 2$ was added, incubated for $10 \mathrm{~min}$ on ice, and centrifuged for $5 \mathrm{~min}$ at 13,000 rpm. The supernatant was used to measure liver TG using ASAN set Triglyceride-S Reagent (Asan Pharmaceutical, Seoul, South Korea) by the enzymatic colorimetric method. Liver lysates and standards were added into the microplate and incubated at $37^{\circ} \mathrm{C}$ for $10 \mathrm{~min}$. Absorbance values were measured at $550 \mathrm{~nm}$ [13].

Protein analysis

As previously described [15], the liver tissue was lysed in $200 \mu$ RIPA buffer. Thereafter, the tissues were homogenized and centrifuged for $30 \mathrm{~min}$ at $14,000 \mathrm{rpm}$. The protein concentration was measured by the BCA method. Samples of equal protein content were resolved by SDS-polyacrylamide gel electrophoresis and transferred to a membrane. The membrane was blocked with $5 \%$ skim milk in PBS. The membrane was subsequently incubated at $4{ }^{\circ} \mathrm{C}$ overnight with primary antibodies against cannabinoid receptor 1 (CB1; sc-293419), Sterol-regulatory element binding protein 1 (SREBP-1; sc-13551), fatty acid synthase (FAS, sc-74540), carnitine palmitoyltransferase1 (CPT1, sc-98834) (all from Santa Cruz Biotechnology, Dallas, TX, USA), AMP-activated protein kinase (AMPKa, \#2532, Cell Signaling Technology, Danvers, MA, USA), and phosphor-AMPK (p-AMPK, \#2531, Cell Signaling Technology, Danvers, MA, USA). The membrane was incubated with its secondary antibody for $1 \mathrm{~h}$ at room temperature. The signal was developed with an ECL solution and visualized with the ImageQuantTM LAS-4000 system (GE Healthcare, Uppsala, Sweden).

Statistical analysis

All statistical analysis was performed with the SPSS 22.0 version (SPSS Inc., Chicago, IL, USA), and the values are reported as means \pm standard error. Two-way repeated measures analysis of variance (ANOVA) was used to compare the changes in body weight, and one-way ANOVA followed by a Tukey HSD post hoc test was conducted to compare the differences between groups after exercise intervention. All data have confirmed the homogeneity of the variance by Levene Statistic, and a significance level of $P$ $=0.05$ was used as a threshold for statistical significance.

\section{Results}

Changes in body weight before and after obesity induction and exercise intervention

Because the body weight in the HF group was a significant increase, mice in the HF group were induced obesity by HFD (Figure 1A). Following 8 weeks of the obesity induction, there was a significant difference across time by group interaction $(F=67.278, p=0.000)$. Post hoc analysis showed that there was a significant increase in body weight in the HF group after 8 weeks of HFD $(t=19.501, p=0.000)$. Besides, the body weight in the HF group was significantly higher than that in the $\mathrm{CO}$ group after 8 weeks of HFD ( $\mathrm{t}$ $=6.000, p=0.000$ ). 
Although HFD induced an increase in body weight, aerobic training prevented an increase in body weight after 8 weeks of exercise intervention (Figure 1B). Following 8 weeks of exercise intervention, there was a significant difference across time by group interaction $(F=8.472, p=0.000)$. Although the body weight in the $\mathrm{CO}$ and HFAT group did not increase, there was a significant increase in the HF $(t=5.374, p=0.000)$ and HFRT group $(t=9.786, p=0.000)$ after 8 weeks of exercise intervention compared to that before. Besides, the body weight in the HF $(p<0.001)$, HFRT $(p<0.001)$, and HFAT $(p<0.01)$ group was significantly higher than that in the CO group after 8 weeks of exercise intervention $(F=12.213, p=$ 0.000).

Changes of liver weight, liver triglycerides, and lipid droplet in liver tissue after exercise intervention

After 8 weeks of exercise intervention, fat mass was significantly higher in the HF group than that in the other groups $(p<0.05$, Figure $2 A)$, and liver weight was significantly higher in the HF $(p<0.05)$ and HFRT $(p<0.05)$ groups than in the CO group (Figure 2B). The size of the lipid droplet of the HF group was larger than that of the $\mathrm{CO}$ group, and even the liver TG of the HF group was significantly higher than that of the CO group $(p<0.01)$ (Figure 2C, 2D). However, the LD size of the training groups decreased, and even the liver TG was not different compared with the $\mathrm{CO}$ group (Figure 2C, 2D).

Protein levels related to fat accumulation in the liver after exercise intervention

Protein levels related to fat accumulation in the liver after 8 weeks of exercise intervention are presented in Figure 3. Following 8 weeks of exercise intervention, CB1 was significantly higher in the HF group than in the $\mathrm{CO}$ group $(\mathrm{p}<0.05)$, and SREBP-1C was significantly higher in the HF group than in the other groups $(p<0.05)$. FAS was significantly higher in the HF and HFRT groups than in the CO and HFAT groups $(p<0.05)$. Following 8 weeks of exercise intervention, $p$-AMPK and $p$-AMPK/AMPK were significantly higher in the training groups than in the HF group $(p<0.05)$, and even the CPT1 was significantly higher in the training groups than in the $\mathrm{CO}$ and HF groups $(p<0.05)$.

\section{Discussion}

The results of this study revealed that 16-week chronic HFD led to a significant increase in body weight and induced an increase of abdominal visceral fat mass and liver steatosis. However, both regular aerobic and resistance exercises were effective in improving fat mass and fat accumulation in the liver.

Regular exercise, along with physical activity and diet, is well-documented, which is the most powerful intervention as a preventive and therapeutic treatment of chronic and adult diseases $[17,18]$. The effect of exercise occurs differently depending on not only in terms of individual characteristics such as age, gender, and disease but also in terms of exercise methods such as frequency, intensity, type, and time $[18,19]$. However, exercise is an intervention without side effects and cost burden against chronic diseases, and the effects of exercise are mainly occurred positively [20]. 
Aerobic exercise mainly improves the cardiovascular system, resulting from an increase of peak oxygen consumption, whereas resistance exercise stimulates the neuromuscular system through repetitive contraction and relaxation of specific muscles [21]. Exercise-induced activation of the cardiovascular and muscular system increases energy consumption during and after exercise. Because where most of the energy needed to produce ATP at rest comes from fatty acid oxidation [22], resulting in contributing to the reduction of body weight and body fat, which is the most powerful effect of exercise [23]. In this study, despite maintaining a high-fat diet, aerobic and resistance exercise inhibited the increase in the amount of adipose tissue. Abdominal visceral fat is a powerful risk factor for chronic inflammation and metabolic disorders that increase insulin resistance and inflammatory levels through the secretion of adipokines $[24,25]$, thus, the reduction of abdominal visceral fat mass induced by exercise could be suggested as a measure of health improvement. As expected, aerobic exercise was effective in suppressing the increase in fat mass in HFD-induced obesity regardless of dietary changes. Although there was no benefit of weight reduction because of no dietary changes to a low-calorie or low-fat diet, the results of this study are noteworthy in that regular resistance exercise also suppresses fat accumulation.

NAFLD is an early stage of liver disease but it is very dangerous in terms of the possibility of metastasis to secondary disease and its involvement in systemic energy metabolism [26]. An increase in sedentary lifestyle and excessive energy intake induced obesity, which easily leads to liver steatosis, therefore, weight loss through lifestyle changes in patients with liver disease has been suggesting as a priority goal for alleviating liver steatosis [9]. For this reason, studies on a dietary conversion [27], and combined treatment of exercise and dietary conversion [28], which are effective in weight loss have been actively studied rather than treatment of exercise alone that is difficult to reduce weight.

The fundamental reason for the occurrence of hepatic steatosis is that an imbalance between fat input and output in the liver has occurred $[29,30]$. If diet regulation is more contributed to reducing the fat input to the liver, exercise could contribute to the fat output by regulating hepatic $\beta$-oxidation and lipogenesis [31]. However, a sudden or quick weight loss through diet changes might lead to a more serious stage of NAFLD [32], and even losing weight is never an easy task for obese people and patients. Therefore, a stepwise approach little by little is important to achieve mitigation of NAFLD through a change in lifestyle. In this aspect, in this study, the existing diet was maintained as a high-fat diet, and moderateintensity aerobic exercise and resistance exercise were conducted. As a result, the aerobic exercise significantly decreased the weight and lipid droplet of the liver, and the resistance exercise decreased the TG and lipid droplet of the liver.

It is well established that aerobic exercise causes lipolysis in various tissues. Aerobic exercise leads to adenosine acetyl-CoA production through the up-regulation of $\beta$-oxidation [33], and the produced AcetylCoA generates ATP in the mitochondrial electron transport system [34]. However, the effector mechanism of the resistance exercise of reducing hepatic steatosis remains unclear. One possible explanation could be that resistance exercise produces intramuscular specific proteins called myokines such as Meteorinlike (Metrnl) and Irisin, and skeletal muscles interact with other organs through the transport of these myokines $[35,36]$. Rao et al. suggested that resistance exercise upregulated peroxisome proliferator- 
activated receptor $y$ coactivator-1a (PGC-1a), resulting in an increase of Metrnl in muscle tissue. And then upregulated Metrnl in skeletal muscle transfers the positive effects of PGC-1a on other tissues [35]. Boström et al. also reported skeletal muscle to interact with other organs by secreting myokine called "Irisin" [36]. Overexpression of intramuscular Irisin reduces hepatic steatosis by inhibiting the lipogenic related factors such as SREBP-1 andFAS in hepatocytes [37]. Therefore, the interaction of muscles with other organs might partially explain the effect of resistance training on the reduction of hepatic steatosis. The results of this study showed that both aerobic and resistance exercise alleviated hepatic steatosis in obese mice by regulating fat accumulation factors in the liver. In this study, muscle-specific proteins were not analyzed, and other organs other than muscle might have interacted with liver tissue. Therefore, future studies will be meaningful to analyze the mechanisms involved in the interaction of hepatic steatosis.

The liver plays an important role as an organ that stores and supplies energy. Liver glycogen is used to continuously replenish the circulating glucose in the blood [38] and is an essential energy source for ATP production in muscles, especially during exercise [39]. Besides, in the liver, glycogen could make up about $5 \%$ of the healthy liver weight [38], sustained exercise is impossible without this action of the liver [40]. In this study, hepatic steatosis was reduced by resistance exercise, but the size and weight of the liver were not decreased. Although the amount of glycogen storage in the liver has not been analyzed, changes to a healthy liver through reduction of hepatic steatosis along with maintenance of liver weight might indicate an improvement of glycogen storage capacity in the liver. Therefore, by analyzing the amount of liver glycogen in future studies, it will be necessary to confirm whether resistance exercise leads to an increase in the storage amount of glycogen in the liver.

In summary, weight reduction was not achieved because there was no dietary conversion to low-fat or low-calorie but both exercises were found to be effective in improving fat mass in adipose tissue and alleviating NAFLD. Moderate-intensity aerobic exercise without dietary restriction improved liver TG and fat accumulation related factors while maintaining body weight and fat mass. Similar to the aerobic exercise, moderate-intensity resistance exercise was effective in improving fat accumulation factors and relieved hepatic steatosis while maintaining liver weight. Therefore, resistance exercise could be recommended for people with obesity or musculoskeletal disorders who have difficulty performing an aerobic exercise for alleviating NAFLD along with aerobic exercise, which was generally recommended.

\section{Conclusion}

The results of this study showed that both aerobic and resistance exercises were effective in improving fat mass and NAFLD even in the absence of weight reduction in HFD-induced obesity. Therefore, a selected sustainable type of exercise based on the fitness level, type of disease, musculoskeletal disorders status, and preference of subjects, will be the best exercise intervention for alleviating NAFLD.

\section{Abbreviations}


NAFLD: Non-alcoholic fatty liver disease

HFD: High-fat diet

CB1: Cannabinoid 1 receptor

SREBP-1 Sterol-regulatory element binding protein 1

FAS: Fatty acid synthase

CPT1: Carnitine palmitoyltransferase1

AMPK: AMP-activated protein kinase

\section{Declarations}

\section{Funding}

This work was supported by the Dong-A University research fund.

\section{Availability of data and materials}

The datasets analyzed during the current study are available from the corresponding author on reasonable request.

\section{Ethics approval}

The animal experiments were approved by the Dong-A University Medical School Institutional Animal Care and Use Committee (DIACUC-approval-19-11)

\section{Consent for publication}

Not applicable.

\section{Competing interests}

The author declares that I have no competing interests.

\section{References}

1. Boden G. Obesity, insulin resistance and free fatty acids. Curr Opin Endocrinol Diabetes Obes. 2011;18:139-43.

2. Torres N, Torre-Villalvazo I, Tovar AR. Nutrigenomics as a Tool in the Prevention of Lipotoxicity: The Case of Soy Protein. Rev Invest Clin. 2019;71:157-67. 
3. Ertunc ME, Hotamisligil GS. Lipid signaling and lipotoxicity in metaflammation: indications for metabolic disease pathogenesis and treatment. J Lipid Res. 2016;57:2099-114.

4. Polyzos SA, Kountouras J, Mantzoros CS. Obesity and Nonalcoholic Fatty Liver Disease: From Pathophysiology to Therapeutics. Metabolism. 2019;92:82-97.

5. Younossi ZM, Koenig AB, Abdelatif D, Fazel Y, Henry L, Wymer M. Global epidemiology of nonalcoholic fatty liver disease-Meta-analytic assessment of prevalence, incidence, and outcomes. Hepatology. 2016;64:73-84.

6. Fan JG, Kim SU, Wong VW. New trends on obesity and NAFLD in Asia. J Hepatol. 2017;67(4):862-73.

7. Haflidadottir S, Jonasson JG, Norland H, Einarsdottir SO, Kleiner DE, Lund SH, Björnsson ES. Longterm follow-up and liver-related death rate in patients with non-alcoholic and alcoholic related fatty liver disease. BMC Gastroenterol. 2014;14:166.

8. Kim D, Li AA, Gadiparthi C, Khan MA, Cholankeril G, Glenn JS, Ahmed A. Changing trends in etiologybased annual mortality from chronic liver disease, from 2007 through 2016. Gastroenterology. 2018;155:1154-63.

9. Chalasani N, Younossi Z, Lavine JE, Charlton M, Cusi K, Rinella M, Harrison SA, Brunt EM, Sanyal AJ. The diagnosis and management of nonalcoholic fatty liver disease: Practice guidance from the American Association for the Study of Liver Diseases. Hepatology. 2018;67:328-57.

10. Patel NS, Doycheva I, Peterson MR, Hooker J, Kisselva T, Schnabl B, Seki E, Sirlin CB, Loomba R. Effect of weight loss on magnetic resonance imaging estimation of liver fat and volume in patients with nonalcoholic steatohepatitis. Clin Gastroenterol Hepatol. 2015;13:561-8.

11. Kwak MS, Kim D. Non-alcoholic fatty liver disease and lifestyle modifications, focusing on physical activity. Korean J Intern Med. 2018;33:64-74.

12. Katsagoni CN, Georgoulis M, Papatheodoridis GV, Panagiotakos DB, Kontogianni MD. Effects of lifestyle interventions on clinical characteristics of patients with non-alcoholic fatty liver disease: A meta-analysis. Metabolism. 2017;68:119-32.

13. Ok DP, Ko K, Bae JY. Exercise without dietary changes alleviates nonalcoholic fatty liver disease without weight loss benefits. Lipids Health Dis. 2018;17:207.

14. Johnson NA, Sachinwalla T, Walton DW, Smith K, Armstrong A, Thompson MW, George J. Aerobic exercise training reduces hepatic and visceral lipids in obese individuals without weight loss. Hepatology. 2009;50:1105-12.

15. Bae JY. Preventive Effects of Different Aerobic Exercise Intensities on the Decline of Cognitive Function in High-Fat Diet-Induced Obese Growing Mice. Medicina. 2020:56:331.

16. Horii N, Uchida M, Hasegawa N, Fujie S, Oyanagi E, Yano H, Hashimoto T, lemitsu M. Resistance training prevents muscle fibrosis and atrophy via down-regulation of $\mathrm{C} 1 \mathrm{q}$-induced Wnt signaling in senescent mice. FASEB J. 2018;32:3547-59.

17. Roberts CK, Barnard RJ. Effects of exercise and diet on chronic disease. J Appl Physiol (1985). 2005;98:3-30. 
18. Pedersen BK, Saltin B. Exercise as medicine - evidence for prescribing exercise as therapy in 26 different chronic diseases. Scand J Med Sci Sports. 2015;25:1-72.

19. Slater GJ, Dieter BP, Marsh DJ, Helms ER, Shaw G, Iraki J. Is an Energy Surplus Required to Maximize Skeletal Muscle Hypertrophy Associated With Resistance Training. Front Nutr. 2019;6:131.

20. Luan X, Tian X, Zhang H, Huang R, Li N, Chen P, Wang R. Exercise as a prescription for patients with various diseases. J Sport Health Sci. 2019;8:422-41.

21. Lambert CP, Evans WJ. Adaptations to aerobic and resistance exercise in the elderly. Rev Endocr Metab Disord. 2005;6:137-43.

22. Speakman JR, Selman C. Physical activity and resting metabolic rate. Proc Nutr Soc. 2003;62(3):62134.

23. Kuo $\mathrm{CH}$, Harris MB. Abdominal fat reducing outcome of exercise training: fat burning or hydrocarbon source redistribution? Can J Physiol Pharmacol. 2016;94:695-8.

24. Dutheil F, Gordon BA, Naughton G, Crendal E, Courteix D, Chaplais E, Thivel D, Lac G, Benson AC. Cardiovascular risk of adipokines: a review. J Int Med Res. 2018;46:2082-95.

25. Feliciano Pereira P, das Graças de Almeida C, Alfenas Rde C. Glycemic index role on visceral obesity, subclinical inflammation and associated chronic diseases. Nutr Hosp. 2014;30:237-43.

26. Paschos P, Paletas K. Non alcoholic fatty liver disease and metabolic syndrome. Hippokratia. 2009;13:9-19.

27. Moosavian SP, Arab A, Paknahad Z. The effect of a Mediterranean diet on metabolic parameters in patients with non-alcoholic fatty liver disease: A systematic review of randomized controlled trials. Clin Nutr ESPEN. 2020;35:40-6.

28. Wong VW, Chan RS, Wong GL, Cheung BH, Chu WC, Yeung DK, Chim AM, Lai JW, Li LS, Sea MM, Chan FK, Sung JJ, Woo J, Chan HL. Community-based lifestyle modification programme for nonalcoholic fatty liver disease: a randomized controlled trial. J Hepatol. 2013;59:536-42.

29. Ferre P, Foufelle F. Hepatic steatosis: A role for de novo lipogenesis and the transcription factor SREBP-1c. Diabetes Obes Metab. 2010;12:83-92.

30. Ferramosca A, Zara V. Modulation of hepatic steatosis by dietary fatty acids. World J Gastroenterol. 2014;20:1746-55.

31. Jeppesen J, Kiens B. Regulation and limitations to fatty acid oxidation during exercise. J Physiol. 2012;590:1059-68.

32. Nseir W, Hellou E, Assy N. Role of diet and lifestyle changes in nonalcoholic fatty liver disease. World J Gastroenterol. 2014;20:9338-44.

33. Hashida R, Kawaguchi T, Bekki M, Omoto M, Matsuse H, Nago T, Takano Y, Ueno T, Koga H, George J, Shiba N, Torimura T. Aerobic vs. resistance exercise in non-alcoholic fatty liver disease: A systematic review. J Hepatol. 2017;66:142-52.

34. Sertie RA, Andreotti S, Proença AR, Campana AB, Lima-Salgado TM, Batista ML, Jr., Seelaender MC, Curi R, Oliveira AC, Lima FB. Cessation of physical exercise changes metabolism and modifies the 
adipocyte cellularity of the periepididymal white adipose tissue in rats. J Appl Physiol (1985). 2013;115:394-402.

35. Rao RR, Long JZ, White JP, Svensson KJ, Lou J, Lokurkar I, Jedrychowski MP, Ruas JL, Wrann CD, Lo JC, Camera DM, Lachey J, Gygi S, Seehra J, Hawley JA, Spiegelman BM. Meteorin-like is a hormone that regulates immune-adipose interactions to increase beige fat thermogenesis. Cell. 2014;157:1279-91.

36. Boström P, Wu J, Jedrychowski MP, Korde A, Ye L, Lo JC, Rasbach KA, Boström EA, Choi JH, Long JZ, Kajimura S, Zingaretti MC, Vind BF, Tu H, Cinti S, Højlund K, Gygi SP, Spiegelman BM. A PGC1-adependent myokine that drives brown-fat-like development of white fat and thermogenesis. Nature. 2012;481:463-8.

37. Park MJ, Kim DI, Choi JH, Heo YR, Park SH. New role of irisin in hepatocytes: The protective effect of hepatic steatosis in vitro. Cell Signal. 2015;27:1831-9.

38. Wasserman DH. Four grams of glucose. Am J Physiol Endocrinol Metab. 2009;296:E11-21.

39. Gonzalez JT, Fuchs CJ, Betts JA, van Loon LJ. Liver glycogen metabolism during and after prolonged endurance-type exercise. Am J Physiol Endocrinol Metab. 2016;311:E543-53.

40. Trefts E, Williams AS, Wasserman DH. Exercise and the Regulation of Hepatic Metabolism. Prog Mol Biol Transl Sci. 2015;135:203-25.

\section{Figures}

A

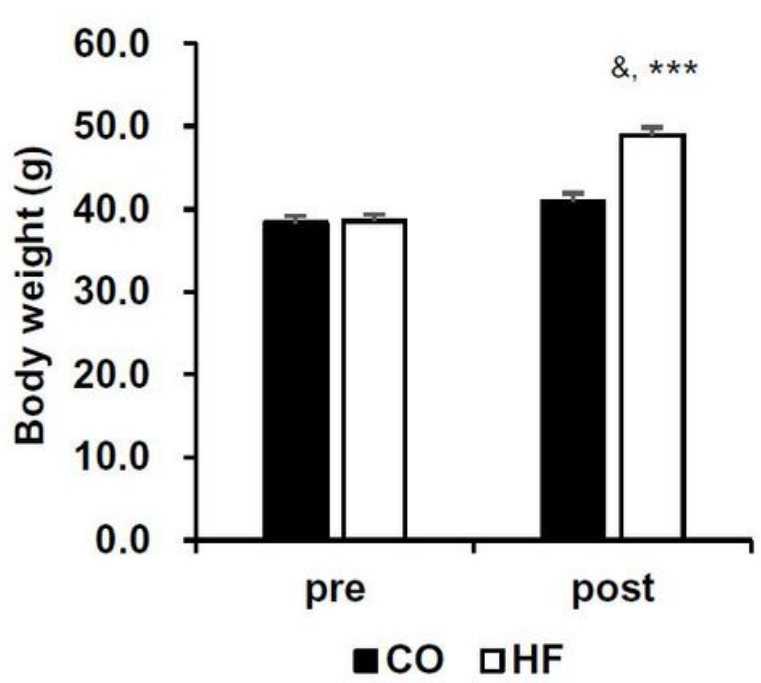

B

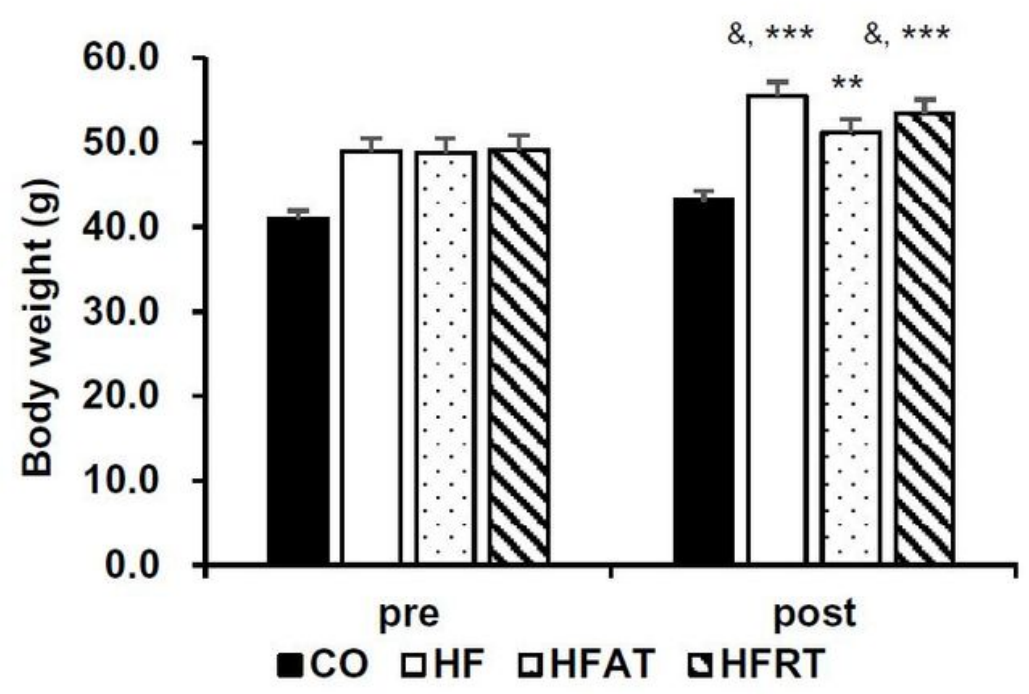

Figure 1

Changes in body weight after obesity induction and exercise intervention Changes in body weight after 8week HFD (A), and 8-week training (B) are presented. Data are expressed as mean \pm SE. CO; Normal-diet 
group, HF; High-fat diet group, HFAT: High-fat diet + Aerobic training group, HFRT: High-fat diet + Resistance training group. \& versus Before, $p<0.001$; $* \star \star$ versus CO group, $p<0.001$; ** versus CO group, $\mathrm{p}<0.01$.
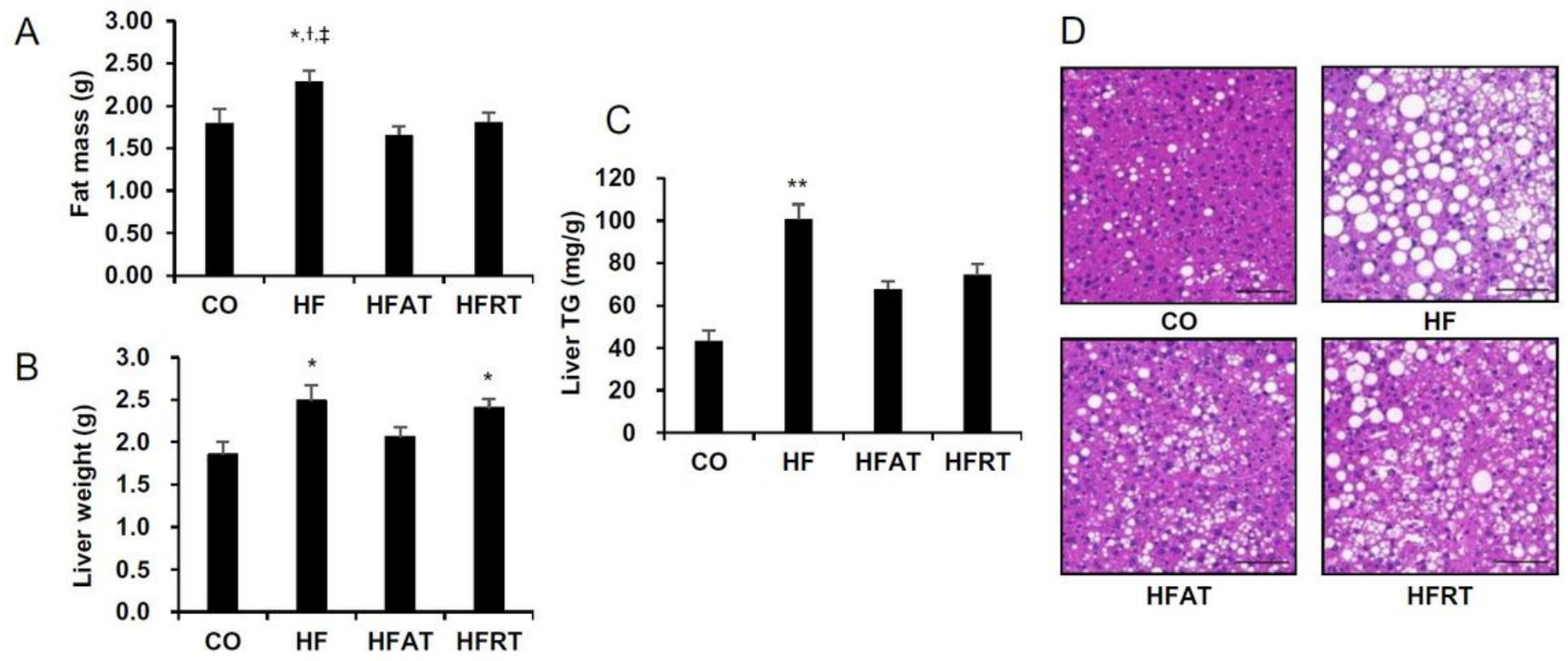

Figure 2

Changes of fat mass, liver weight, liver triglycerides, and lipid droplet in liver tissue after exercise intervention Changes in fat mass (A), liver weight (B), triglycerides (C), and lipid droplet (D) in the liver after exercise intervention are presented. Data are expressed as mean $\pm \mathrm{SE}$. CO; Normal-diet group, HF; High-fat diet group, HFAT: High-fat diet + Aerobic training group, HFRT: High-fat diet + Resistance training group. * versus CO group, $p<0.05$; ** versus CO group, $p<0.01 ; \uparrow$ versus HFAT group, $p<0.05 ; \ddagger$ versus HFRT group, $p<0.05$. Scale bar $=20$ um. 

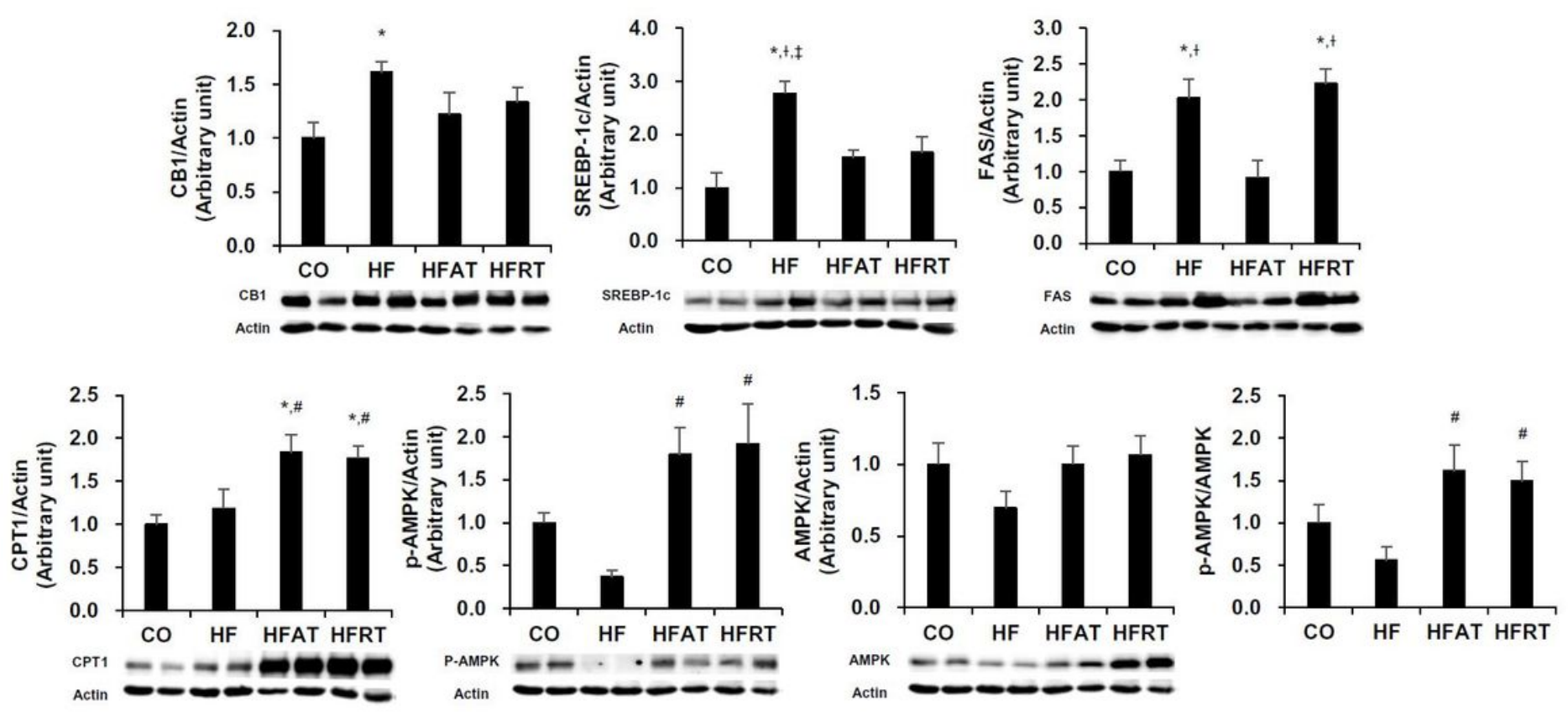

Figure 3

Protein levels related to fat accumulation in the liver after exercise intervention Data are expressed as mean \pm SE. CO; Normal-diet group, HF; High-fat diet group, HFAT: High-fat diet + Aerobic training group, HFRT: High-fat diet + Resistance training group. * versus CO group, $p<0.05$; \# versus HF group, $p<0.05$; $†$ versus HFAT group, $p<0.05$; $\ddagger$ versus HFRT group, $p<0.05$. 